\title{
ZIF-8-Derived Hollow Carbon for Efficient Adsorption of Antibiotics
}

\author{
Hongmei Tang ${ }^{1}$, Wenyao $\mathrm{Li}^{1}{ }^{1} * \mathbb{C}$, Haishun Jiang ${ }^{1}$, Runjia Lin ${ }^{2}$, Zhe Wang ${ }^{1}$, Jianghong $\mathrm{Wu}^{3}$,

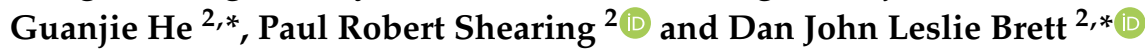 \\ 1 School of Materials Engineering, Shanghai University of Engineering Science, Shanghai 201620, China; \\ neview2015@163.com (H.T.); jhaishun@163.com (H.J.); 18317188857@163.com (Z.W.) \\ 2 Electrochemical Innovation Lab, Department of Chemical Engineering, University College London, \\ London WC1E 7JE, UK; runjia.lin.16@ucl.ac.uk (R.L.); p.shearing@ucl.ac.uk (P.R.S.) \\ 3 College of Health Science and Environmental Engineering, Shenzhen Technology University, \\ Shenzhen 518118, China; wujianghong@sztu.edu.cn \\ * Correspondence: liwenyao314@gmail.com (W.L.); g.he@ucl.ac.uk (G.H.); d.brett@ucl.ac.uk (D.J.L.B.)
}

Received: 4 December 2018; Accepted: 15 January 2019; Published: 18 January 2019

\begin{abstract}
The harmful nature of high concentrations of antibiotics to humans and animals requires the urgent development of novel materials and techniques for their absorption. In this work, CTAB (Cetyltrimethyl Ammonium Bromide)-assisted synthesis of ZIF-8 (zeolitic imidazolate framework)-derived hollow carbon (ZHC) was designed, prepared, and used as a high-performance adsorbent, and further evaluated by Langmuir and Freundlich isothermal adsorption experiments, dynamic analysis, as well as theoretical calculation. The maximum capacities of ZHC for adsorbing tetracycline (TC), norfloxacin (NFO), and levofloxacin (OFO) are 267.3, 125.6, and $227.8 \mathrm{mg} \mathrm{g}^{-1}$, respectively, which delivers superior adsorptive performance when compared to widely studied inorganic adsorbates. The design concept of ZIF-8-derived hollow carbon material provides guidance and insights for the efficient adsorbent of environmental antibiotics.
\end{abstract}

Keywords: ZIF-8; hollow carbon; antibiotics; adsorbent

\section{Introduction}

Due to their outstanding medical properties, antibiotics are broadly utilized to treat infectious diseases; this leads to high levels of antibiotics in hospital effluent and sewage treatment plants [1]. Since antibiotics cannot be totally metabolized by humans or animals, and it is impossible to prevent the mass utilization of antibiotics, a vicious cycle forms and results in the uncontrolled accumulation of residual antibiotics in the environment [2]. Antibiotics such as quinolones and tetracycline are able to make bacteria resistant to drugs and cause various diseases. Therefore, with the purpose of preventing the pollution of antibiotics, materials with tremendous absorption ability for antibiotics are urgently required.

In recent years, metal-organic frameworks (MOFs) have been synthesized via compounds composed of inorganic metal ions centers and organic ligands [3]. A great number of targeted applications can be achieved through the application of MOFs, owing to their diversity, as they are composed of various metal ions and organic ligands. Additionally, various morphologies of porous nanomaterials can be prepared by using MOFs as sacrificial templates through different thermal and/or chemical treatments. For instance, highly porous carbons can be produced via the heat treatment of MOFs in an inert atmosphere, coupled with chemical etching for the removal of the surface metal ions, thus, leading to an increased specific surface area. Compared with carbonaceous materials fabricated by conventional precursors, MOF-derived carbons often exhibit controllable porous architectures, pore 
volumes, and surface areas [4]. Various porous carbon materials, with controlled morphologies from 0 to 3 dimensions, have been successfully derived through the carbonization of MOFs, highlighting their versatility as precursors [5-7]. In addition to porous materials, MOF-derived carbons can be converted into hollow structures.

In this work, CTAB (Cetyltrimethyl Ammonium Bromide)-assisted synthesis of ZIF-8, a subclass of MOFs, was applied as a sacrificial template to synthesize ZIF-8 (zeolitic imidazolate framework)-derived hollow carbon (ZHC) nanostructures, with the aim of exploring highly efficient absorbent materials. The maximum adsorption capacities of ZHC for tetracycline (TC), norfloxacin (NFO), and levofloxacin (OFO) are investigated according to the data obtained from Langmuir and Freundlich isothermal adsorption simulation, as well as kinetic experiments. The adsorptive performance of $\mathrm{ZHC}$ is comparable to the cutting-edge inorganic adsorbents and paves a new way for the design concept of adsorbents.

\section{Experimental}

\subsection{Chemicals}

Zinc nitrate hexahydrate $\left(\mathrm{Zn}\left(\mathrm{NO}_{3}\right)_{2} \cdot 6 \mathrm{H}_{2} \mathrm{O}\right)$, tannic acid (TA) and cetyltrimethylammonium bromide (CTAB, analytical purity) were all purchased from Sinopharm Chemical Reagent Co., Ltd. (Shanghai, China). Anhydrous methanol (AR) and hydrochloric acid ( $\mathrm{HCl}, 36 \%)$ were purchased from Shanghai Lingfeng Chemical Reagent Co., Ltd. (Shanghai, China). 2-methylimidazole (AR, 98\%) was purchased from in Aladdin Industrial Corporation (Shanghai, China).

\subsection{ZIF-8 Derived Hollow Carbon (ZHC) Preparation}

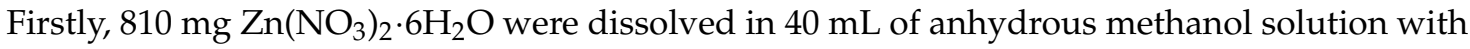
continuous stirring, which was named Solution A. $526 \mathrm{mg}$ of 2-methylimidazole were added into $40 \mathrm{~mL}$ of anhydrous methanol solution with continuous stirring until a homogeneous solution formed, which was named Solution B. Then, Solution A and B were mixed and a further $10 \mathrm{mg}$ of CTAB was added, the mixture was sonicated for $10 \mathrm{~min}$ then sealed for $24 \mathrm{~h}$ at room temperature. The obtained white ZIF-8 products were washed with anhydrous methanol several times. After drying, the products were transferred to a $40 \mathrm{~mL}$ tannic acid solution $\left(0.01 \mathrm{~mol} \mathrm{~L}^{-1}\right)$, sonicated for $5 \mathrm{~min}$, then collected by centrifugation and washed with water and methanol successively. After drying, the samples were put into a tube furnace and heated at $800{ }^{\circ} \mathrm{C}$ for $2 \mathrm{~h}$ under $\mathrm{N}_{2}$ atmosphere. Finally, the collected black powders were immersed in a $1 \mathrm{~mol} \mathrm{~L}^{-1}$ of $\mathrm{HCl}$ solution to remove the residual $\mathrm{Zn}$ or $\mathrm{ZnO}$. Finally, the samples were dried under vacuum at $100^{\circ} \mathrm{C}$ to obtain the final products after washing with deionized water several times. A simple schematic illustration of this process is shown in Figure 1.
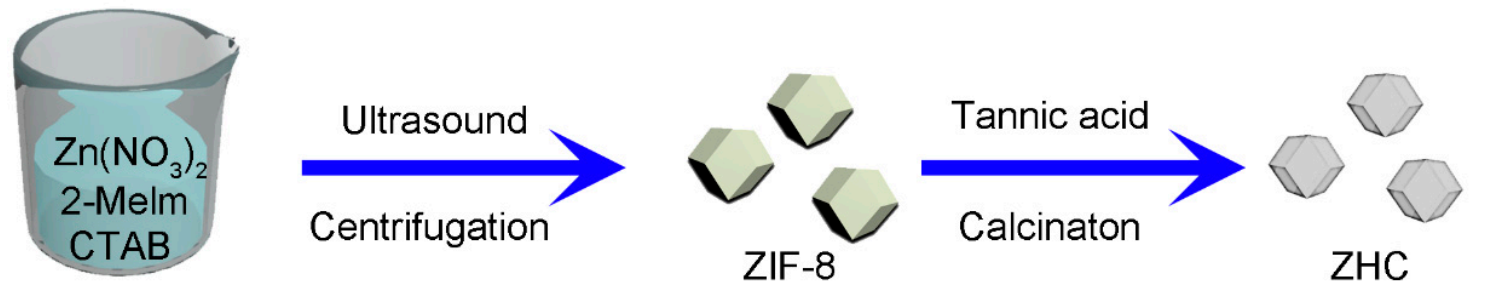

Figure 1. Schematic illustration of the fabrication process of ZIF-8 (zeolitic imidazolate framework)-derived hollow carbon (ZHC).

\subsection{Characterization}

The microstructure of the material was characterized by scanning electron microscopy (SEM, S-4800, Hitachi, Japan). Transmission electron microscopy (TEM) analysis was performed using a JEM-2100F system (Rigaku, Japan). The X-ray diffraction (XRD) was carried out with a $\mathrm{Cu} \mathrm{K} \alpha$ radiation source (Rigaku, Japan). The Brunauer-Emmett-Teller (BET) method was used to provide the specific 
surface area value. The pore size distribution of the $\mathrm{ZHC}$ was obtained from $\mathrm{BJH}$ method using the Density Functional Theory (DFT) method by ASiQwin software [8,9] (ASAP 2020, Micromeritics, Norcross, GA, USA).

\subsection{Adsorption Process}

The as-prepared ZHC can be applied as an efficient adsorbent for antibiotics in water. The antibiotics solution with increasing concentrations (TC, OFO 5-40 $\mathrm{mg} \mathrm{L}^{-1}$, NFO 4-20 mg L ${ }^{-1}$, there chemical structures are shown in Figure S1) were carried out in a $100 \mathrm{~mL}$ conical flask with $5 \mathrm{mg}$ ZHC; the adsorption begins when the solution is mixed with the absorbent. The adsorption experiments of three antibiotics were carried out at a constant speed (1000 rpm) in order to ensure the uniform diffusion of the absorbent in the solution. The different initial concentrations supernatant solution was obtained by filtering the solution with a syringe through a water filter membrane of $0.22 \mu \mathrm{m}$ (SHZ-D (III) circulating water vacuum pump). After filtering, UV-vis spectra of the supernatant solution were recorded at a characteristic wavelength. A batch of adsorption experiments and blank experiments were conducted under constant temperature and $\mathrm{pH}$ value. The maximum adsorption amount of $\mathrm{ZHC}$ for TC, OFO and NFO is calculated using the formula (1):

$$
q_{e}=\frac{\left(C_{0}-C_{e}\right) V}{m}
$$

where $q_{e}(\mathrm{mg} / \mathrm{g})$ represents the absorption capacity of $\mathrm{ZHC}, C_{0}(\mathrm{mg} / \mathrm{L})$ represents the initial concentration of TC/OFO/NFO, $C_{e}(\mathrm{mg} / \mathrm{L})$ refers to the concentration of TC/OFO/NFO when the solution reaches the adsorption equilibrium, while $V(\mathrm{~L})$ represents the solution volume and $m(\mathrm{~g})$ represents the mass of the absorbent $\mathrm{ZHC}$.

The adsorption of ZHC for antibiotics is based on Langmuir and Freundlich isothermal adsorption models. The Langmuir model formula $[10,11]$ is as follows:

$$
\frac{C_{e}}{q_{e}}=\frac{1}{K_{L} q_{m}}+\frac{C_{e}}{q_{m}}
$$

where $C_{e}$ is the equilibrium concentration, $K_{L}\left(\mathrm{~L} \mathrm{mg}^{-1}\right)$ is the adsorption constant, and $q_{m}(\mathrm{mg} / \mathrm{g})$ is the maximum adsorption capacity.

The Freundlich model formula $[7,12]$ is as follows:

$$
\ln q_{e}=\ln K_{f}+\left(\frac{1}{\mathrm{n}}\right) \ln C_{e}
$$

where $K_{f}\left(\left(\mathrm{mg} \mathrm{g}^{-1}\right) \times\left(\mathrm{L} \mathrm{mg}^{-1}\right)^{1 / \mathrm{n}}\right)$ and $\mathrm{n}$ represent the Freundlich constant. The higher the value, the better the adsorption effect.

The pseudo-first-order adsorption kinetics model of ZHC for antibiotics assumes that adsorption is controlled by diffusion steps and is given as [13]:

$$
\ln \left(q_{e}-q_{t}\right)=\ln q_{e}-K_{1} t
$$

where $q_{t}\left(\mathrm{mg} \mathrm{g}^{-1}\right)$ represents the amount of antibiotics absorbed in time $t(\mathrm{~min})$ and $K_{1}\left(\mathrm{~min}^{-1}\right)$ represents the first-order kinetic constant.

The pseudo-second-order kinetic model assumes that the adsorption rate is determined by the square value of the number of unoccupied adsorptive vacancies on the adsorbent surface, and the adsorption process is controlled by the chemical adsorption mechanism. The pseudo-second-order kinetic model $[13,14]$ is as follows:

$$
\frac{t}{q_{t}}=\frac{1}{K_{2} q_{e}^{2}}+\frac{t}{q_{e}}
$$


where $K_{2}\left(\mathrm{~g} \mathrm{mg}^{-1} \mathrm{~min}^{-1}\right)$ represents the pseudo-second-order kinetic constant.

The formula of the intra-particle diffusion model $[15,16]$ is as follows:

$$
q_{t}=K_{i d} t^{\frac{1}{2}}+C
$$

where $C\left(\mathrm{mg} \mathrm{g}^{-1}\right)$ is the constant involving the thickness, the boundary layer and $K_{i d}\left(\mathrm{mg} \mathrm{g}^{-1} \mathrm{~min}^{-1 / 2}\right)$ is the internal diffusion constant. In the particle diffusion model, $q_{t}$ and $t^{1 / 2}$ are linearly fitted. If the straight line passes the origin, it is shown that the diffusion of the particles is the rate-limiting step of the adsorption process, and the adsorption process is controlled by the other adsorption stages if the origin is not passed.

\section{Results and Discussion}

To investigate the crystal structure of the obtained ZHC, XRD analysis was conducted. As shown in Figure 2a, the diffraction peaks $(2 \theta)$ located at $26.61^{\circ}, 43.45^{\circ}$, and $46.32^{\circ}$ can be attributed to the (111), (100), and (110) crystal faces of graphite, respectively; which is in accordance with the peaks displayed in the graphite standard (JCPDS No. 01-075-2078). No impurity peak was found in the XRD pattern, demonstrating the high purity of the obtained ZHC materials. The morphology of ZIF-8 (Figure S2) and ZHC were explored by SEM. During the synthesis of ZIF-8, the addition of CTAB will significantly reduce the particle size of ZIF-8 crystals. It is speculated that CTAB may control the growth of crystals during synthesis, and the growth control mode may be as follows: Because of the anisotropic growth of ZIF-8 itself, part of CTAB is selectively adsorbed to the lowest level on the plane of mutual acting force, when $C T A B$ is added into the precursor solution, CTAB in the process is more likely to act as a capping agent, because CTAB can be absorbed by the ZIF- 8 crystals on the surface [17,18]. Therefore, the growth and separation of ZIF- 8 crystals can be inhibited, and the morphology and size of ZIF-8 nanoparticles can be further controlled. According to the SEM images presented in Figure $2 b, c$, the particle size of the ZIF material witnessed a slight decrease after carbonization, which can be explained by the shrinkage of organic components during the calcination process. The TEM image displayed in Figure $2 \mathrm{~d}$ confirms that the ZHC materials show obvious hollow structures.
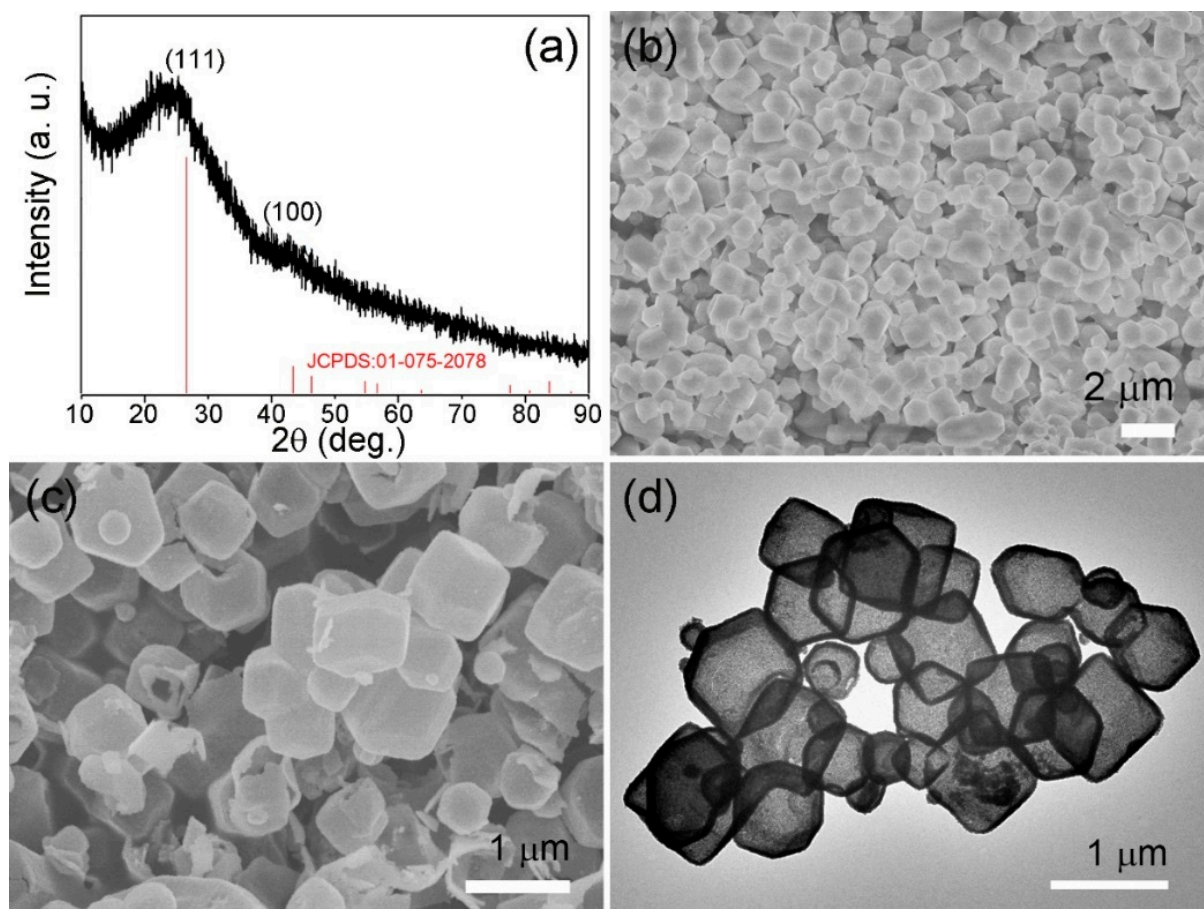

Figure 2. (a) X-ray diffraction (XRD) pattern of the ZHC; (b,c) scanning electron microscopy (SEM) image of ZIF-8 and ZHC; (d) transmission electron microscopy (TEM) image of the obtained ZHC. 
$\mathrm{N}_{2}$ adsorption-desorption isotherms were carried out to investigate the pore diameter distribution and the specific surface area of ZHC. It can be seen from Figure 3a that the relative pressure $\left(P / P_{0}\right)$ between $0.2-0.9$ shows a gradual upward trend of nitrogen adsorption and the pore diameter is concentrated at $5 \mathrm{~nm}$ (Figure 3b), which indicates that a mesoporous structure exists in the ZHC material. The adsorbent with a higher surface area $\left(807.56 \mathrm{~m}^{2} \mathrm{~g}^{-1}\right)$ can enhance the adsorption capacity of the adsorbent due to its abundant active adsorption sites. In addition, the mesoporous structure could give rise to the ability and efficiency of removing antibiotics pollutants [19].
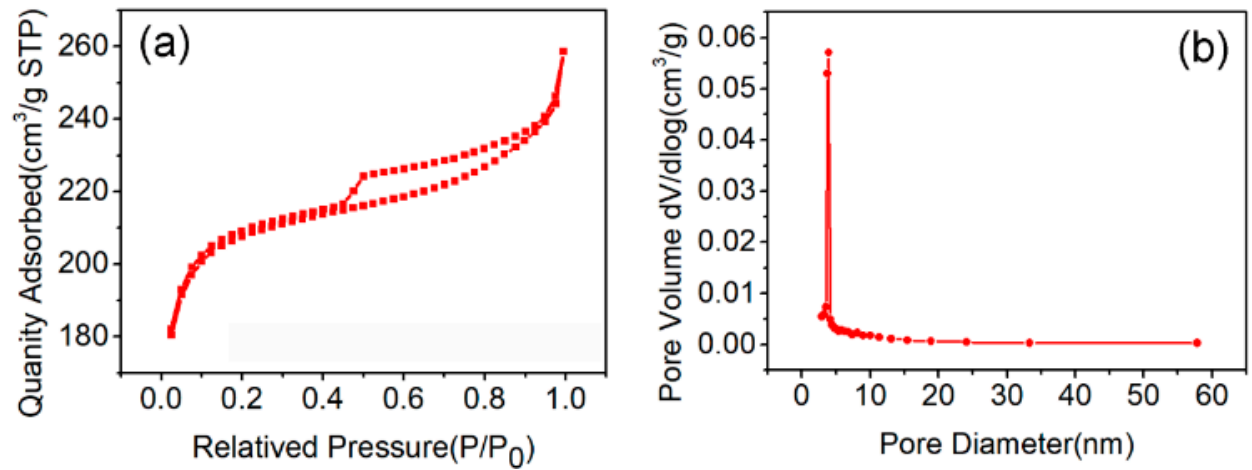

Figure 3. (a) $\mathrm{N}_{2}$ adsorption-desorption isotherm; and (b) pore size distribution of $\mathrm{ZHC}\left(\mathrm{N}_{2} 77 \mathrm{~K}, \mathrm{DFT}\right)$.

According to the data in Table 1, the adsorption process of the $\mathrm{ZHC}$ on TC, NFO, and OFO is in accordance with the Langmuir (Figure $4 a, b$ ) and Freundlich (Figure 4c,d) models. However, considering the correlation fitting coefficient, the Langmuir model can describe the adsorption process well; that is, the adsorption of the material on TC, NFO, and OFO is homogeneous in the single molecular layer. The fitting of experimental data to model equations based on the Langmuir model shows the adsorption capacity of the ZHC on TC, NFO, and OFO are 267.3, 125.6, and $227.8 \mathrm{mg} \mathrm{g}^{-1}$, respectively.
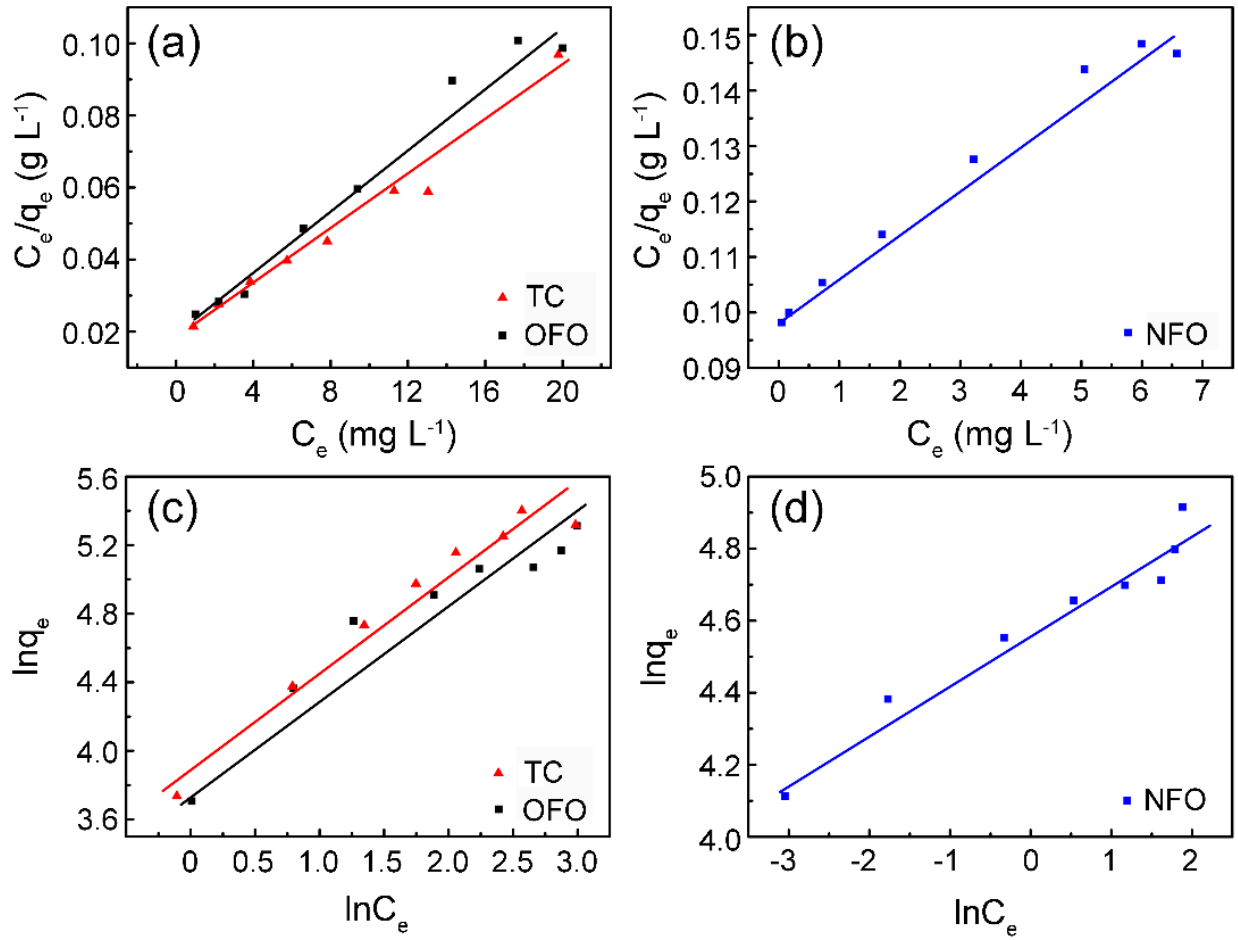

Figure 4. The isothermal adsorption fitting curves of Langmuir $(\mathbf{a}, \mathbf{b})$ and Freundlich (c,d) for tetracycline (TC), levofloxacin (OFO), and norfloxacin (NFO) by ZHC. 
Table 1. The relevant fitting parameters of tetracycline (TC), norfloxacin (NFO), and levofloxacin (OFO) simulated using Langmuir and Freundlich models.

\begin{tabular}{|c|c|c|c|c|c|c|}
\hline \multirow[b]{2}{*}{ Pollutants } & \multicolumn{3}{|c|}{ Langmuir } & \multicolumn{3}{|c|}{ Freundlich } \\
\hline & $\begin{array}{c}K_{L} \\
\left(\mathrm{~L} \mathrm{mg}^{-1}\right)\end{array}$ & $\begin{array}{c}q_{m} \\
\left(\mathrm{mg} \mathrm{g}^{-1}\right)\end{array}$ & $R^{2}$ & $\begin{array}{cl} & K_{f} \\
\left(\mathrm{mg} \mathrm{g}^{-1}\right) & \left(\mathrm{L} \mathrm{mg}^{-1}\right)^{1 / n}\end{array}$ & $n$ & $R^{2}$ \\
\hline $\mathrm{TC}$ & 0.212 & 267.3 & 0.972 & 70.80 & 1.8319 & 0.948 \\
\hline NFO & 0.079 & 125.6 & 0.979 & 53.41 & 7.23 & 0.945 \\
\hline OFO & 0.230 & 227.8 & 0.973 & 26.08 & 2.114 & 0.91 \\
\hline
\end{tabular}

As can be found in Table 2, the ZHC exhibits much higher antibiotics adsorption ability than pure ZIF-8 (Figure S3) and several inorganic materials in the reported literature, demonstrating the huge potential of the $\mathrm{ZHC}$ in adsorption applications.

Table 2. Comparison of the TC, NFO, and OFO adsorption ability of ZHC with other inorganic adsorbents.

\begin{tabular}{|c|c|c|c|c|}
\hline Antibiotics & Adsorbents & $q_{m}\left(\mathrm{mg} \mathrm{g}^{-1}\right)$ & Condition $(\mathrm{pH})$ & Reference \\
\hline \multirow[t]{6}{*}{ Tetracycline } & GN & $2 \times 10^{-4}$ & 7 & [20] \\
\hline & $\mathrm{E}_{3} \mathrm{D}_{7}$ & 133.3 & 8 & [21] \\
\hline & $\mathrm{CNT}-2 \% \mathrm{O}$ & 217.8 & 4 & [22] \\
\hline & Biochar & 102 & 6 & [23] \\
\hline & ZIF-8 & 119.04 & 7 & This work \\
\hline & $\mathrm{ZHC}$ & 267.3 & 7 & This work \\
\hline \multirow[t]{4}{*}{ Norfloxacin } & RGOS & 50 & 6 & [24] \\
\hline & H-CNTS & 76.3 & 7 & [25] \\
\hline & ZIF-8 & 38.69 & 7 & This work \\
\hline & $\mathrm{ZHC}$ & 125.6 & 7 & This work \\
\hline \multirow[t]{5}{*}{ Ofloxacin } & GN & 0.2 & 7 & [20] \\
\hline & BEPS-free biofilm-50 & 5.27 & 7 & [26] \\
\hline & Cassava residue-derived biochar & 3.00 & 7 & [27] \\
\hline & ZIF-8 & 111.48 & 7 & This work \\
\hline & $\mathrm{ZHC}$ & 227.8 & 7 & This work \\
\hline
\end{tabular}

Figure 5a shows that the adsorption of the $\mathrm{ZHC}$ to the three antibiotics reached equilibrium after $\sim 48 \mathrm{~h}$. Figure $5 \mathrm{~b}, \mathrm{c}$ are the fitted curves of pseudo-first-order dynamic models and pseudo-second-order dynamic models, respectively. Considering the correlation coefficient $R^{2}$ values in Table 3, it can be concluded that the adsorption of the ZHC to the three antibiotics is more in line with the pseudo-second-order kinetic model, meaning the chemical adsorption mechanism exists in the adsorption process [7]. Figure 5d depicts the fitting curve of intra-particle diffusion model, which indicates that the adsorption process of the antibiotics can be divided into three stages: (1) antibiotics diffuse on the surface of the adsorbent; (2) antibiotics penetrate in the inner structure of the ZHC through pores; (3) antibiotics interact with the surface-active sites of the ZHC. The initial phase of stage (1) is regarded as the diffusion of antibiotics through the solution to the exterior surface of the ZHC, which can be known as external diffusion. Stage (2) shows the gradual adsorption where intraparticle diffusion is rate-limiting. Stage (3) is very fast and cannot be treated as the rate-controlling step, which is considered negligible. The regressions of $q_{t}$ vs. $t^{1 / 2}$ for the antibiotic solutions were linear and did not pass through the origin, which means that the boundary layer diffusions were in control during the adsorption process and the intra-particle diffusions were involved in the adsorption process. 

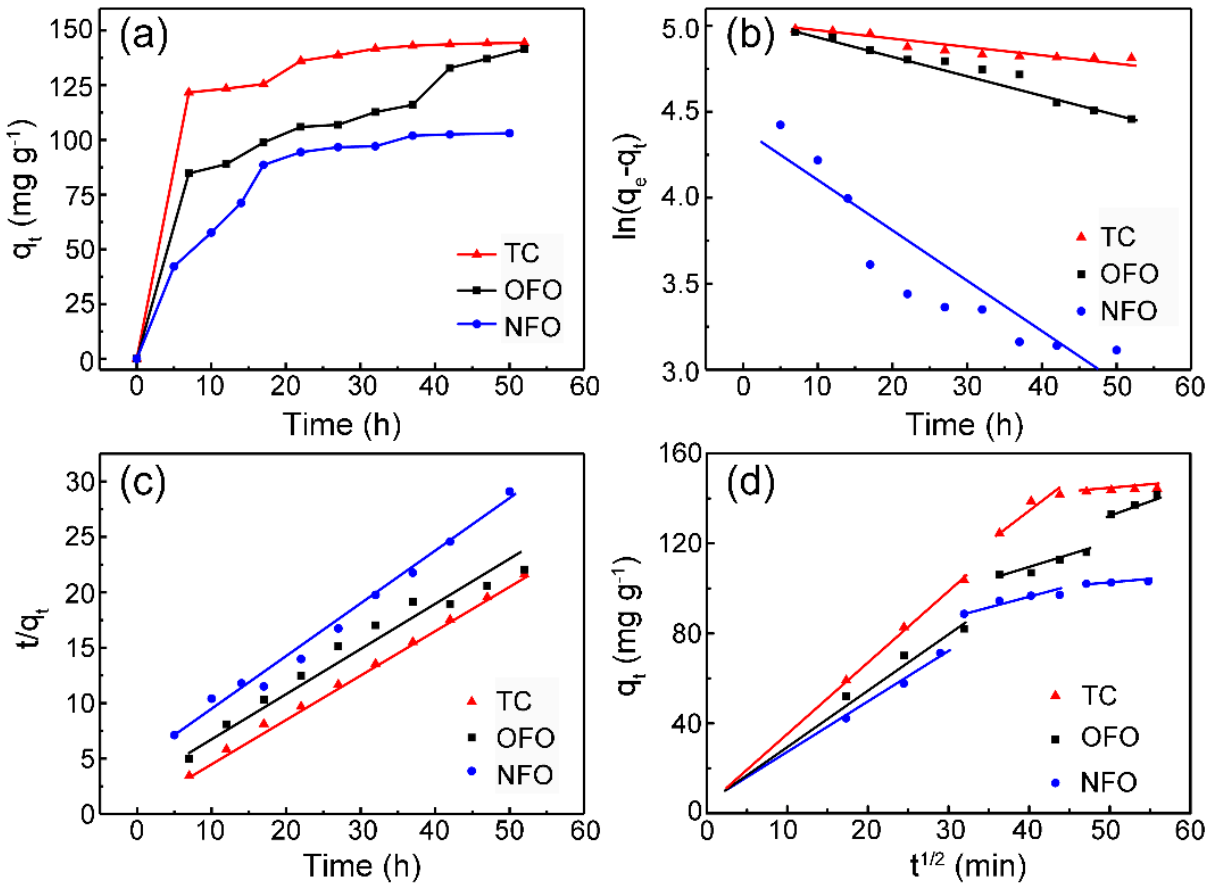

Figure 5. (a) Time curve fitting; (b) pseudo-first-order kinetic model; (c) pseudo-second-order kinetic model; and (d) the intra-particle diffusion model of ZHC.

Table 3. The related fitting parameters of the kinetic model.

\begin{tabular}{|c|c|c|c|c|c|c|c|}
\hline \multirow[b]{2}{*}{ Model } & \multirow{2}{*}{$\begin{array}{c}C_{0} \\
\left(\mathrm{mg} \mathrm{L}^{-1}\right)\end{array}$} & \multicolumn{3}{|c|}{ Pseudo-First-Order } & \multicolumn{3}{|c|}{ Pseudo-Second-Order } \\
\hline & & $\begin{array}{c}K_{1} \\
\left(\mathrm{~L} \mathrm{~min}^{-1}\right)\end{array}$ & $\begin{array}{c}q_{e, c a l} \\
\left(\mathrm{mg} \mathrm{g}^{-1}\right)\end{array}$ & $R^{2}$ & $\left(\mathrm{~g} \mathrm{mg}^{-1} \min ^{-1}\right)$ & $\begin{array}{c}q_{e, c a l} \\
\left(\mathrm{mg} \mathrm{g}^{-1}\right)\end{array}$ & $R^{2}$ \\
\hline $\mathrm{TC}$ & 40 & 0.00422 & 148.21 & 0.852 & $4.33 \times 10^{-5}$ & 151.89 & 0.998 \\
\hline NFO & 20 & 0.02963 & 76.77 & 0.87 & $6.25 \times 10^{-5}$ & 126.17 & 0.987 \\
\hline $\mathrm{OFO}$ & 40 & 0.01136 & 158.92 & 0.954 & $3.83 \times 10^{-5}$ & 161.60 & 0.961 \\
\hline
\end{tabular}

\section{Conclusions}

In summary, a novel ZIF-8-derived hollow carbon (ZHC) was developed as an efficient adsorbent to remove the residual antibiotics in the polluted water. The strong adsorption capacity was confirmed by Langmuir and Freundlich isothermal adsorption experiments, dynamic analysis, as well as theoretical calculation. The maximum quantities of $\mathrm{ZHC}$ on adsorbing tetracycline (TC), norfloxacin (NFO), and levofloxacin (OFO) are 267.3, 125.6, and $227.8 \mathrm{mg} \mathrm{g}^{-1}$, respectively. The excellent adsorptive performance of ZHC could be attributed to the hollow structure, which endows its high BET surface area and sufficient adsorption sites. Our results suggested that the MOF-derived carbon-material provides an extensible method to efficiently remove the antibiotics in the environment.

Supplementary Materials: The following are available online at http:/ /www.mdpi.com/2079-4991/9/1/117/s1, Figure S1: The chemical structure of TC, NFO and OFO. Figure S2: The Low-(a) and High-magnification (b) SEM images of the synthesized ZIF-8 without CTAB. Figure S3: The isothermal adsorption fitting curves of Langmuir $(\mathrm{a}, \mathrm{b})$ and Freundlich $(\mathrm{c}, \mathrm{d})$ for TC, OFO and NFO by ZIF-8.

Author Contributions: W.L. and G.H. conceived and designed the experiments, H.T., H.J., R.L., Z.W., J.W., P.R.S. performed the experiments and analyzed the data, H.T., W.L., G.H. and D.J.L.B. wrote the manuscript, W.L., G.H. and D.J.L.B. supervised the whole project and acquired funding. All the authors revised and checked draft.

Funding: The work has been funded by the National Natural Science Foundation of China (51602193, 21601122, 51701022), Shanghai “Chen Guang" project (16CG63), the Fundamental Research Funds for the Central Universities (WD1817002), and the Talent Program of Shanghai University of Engineering Science, Science and Technology Facilities Council for STFC/MDC Futures Early Career Award, and Engineering and Physical Sciences Research Council (EPSRC) (EP/R023581/1). 
Conflicts of Interest: The authors declare no conflict of interest.

\section{References}

1. Michael, I.; Rizzo, L.; McArdell, C.S.; Manaia, C.M.; Merlin, C.; Schwartz, T.; Dagot, C.; Fatta-Kassinos, D. Urban wastewater treatment plants as hotspots for the release of antibiotics in the environment: A review. Water Res. 2013, 47, 957-995. [CrossRef] [PubMed]

2. Liu, M.K.; Liu, Y.Y.; Bao, D.D.; Zhu, G.; Yang, G.H.; Geng, J.F.; Li, H.T. Effective Removal of Tetracycline Antibiotics from Water using Hybrid Carbon Membranes. Sci. Rep. UK 2017, 7, 43717. [CrossRef] [PubMed]

3. Zhou, H.C.; Long, J.R.; Yaghi, O.M. Introduction to Metal-Organic Frameworks. Chem. Rev. 2012, 112, 673-674. [CrossRef] [PubMed]

4. Chaikittisilp, W.; Ariga, K.; Yamauchi, Y. A new family of carbon materials: Synthesis of MOF-derived nanoporous carbons and their promising applications. J. Mater. Chem. A 2013, 1, 14-19. [CrossRef]

5. Xu, H.; Zhou, S.; Xiao, L.; Wang, H.; Li, S.; Yuan, Q. Fabrication of a nitrogen-doped graphene quantum dot from MOF-derived porous carbon and its application for highly selective fluorescence detection of $\mathrm{Fe}^{3+}$. J. Mater. Chem. C 2015, 3, 291-297. [CrossRef]

6. Pachfule, P.; Shinde, D.; Majumder, M.; Xu, Q. Fabrication of carbon nanorods and graphene nanoribbons from a metal-organic framework. Nat. Chem. 2016, 8, 718-724. [CrossRef] [PubMed]

7. Xia, W.; Qiu, B.; Xia, D.; Zou, R. Facile preparation of hierarchically porous carbons from metal-organic gels and their application in energy storage. Sci. Rep. UK 2013, 3, 1935. [CrossRef]

8. Thommes, M.; Kaneko, K.; Neimark, A.V.; Olivier, J.P.; Rodriguez-Reinoso, F.; Rouquerol, J.; Sing, K.S.W. Physisorption of gases, with special reference to the evaluation of surface area and pore size distribution (IUPAC Technical Report). Pure Appl. Chem. 2015, 87, 1051-1069. [CrossRef]

9. Francisco, J.S.; Katie, A.C.; Matthias, T. Characterization of Micro/Mesoporous Materials by Physisorption: Concepts and Case Studies. Acc. Mater. Surf. Res. 2018, 3, 34-50.

10. Li, W.; Wang, J.; He, G.; Yu, L.; Noor, N.; Sun, N.; Zhou, X.; Hu, J.; Parkin, I.P. Enhanced adsorption capacity of ultralong hydrogen titanate nanobelts for antibiotics. J. Mater. Chem. A 2017, 5, 4352-4358. [CrossRef]

11. Langmuir, I. The adsorption of gases on plane surfaces of glass, mica and platinum. J. Am. Chem. Soc. 1918, 40, 1361-1403. [CrossRef]

12. Freundlich, H.M.F. Over the adsorption in solution. J. Phys. Chem. 1906, 57, 385-470.

13. Dai, J.; Xiao, X.; Duan, S.; Liu, J.; He, J.; Lei, J.; Wang, L. Synthesis of novel microporous nanocomposites of ZIF-8 on multiwalled carbon nanotubes for adsorptive removing benzoic acid from water. Chem. Eng. J. 2018, 331, 64-74. [CrossRef]

14. Ho, Y.-S. Review of second-order models for adsorption systems. J. Hazard. Mater. 2006, 136, 681-689. [CrossRef] [PubMed]

15. Singh, S.K.; Townsend, T.G.; Mazyck, D.; Boyer, T.H. Equilibrium and intra-particle diffusion of stabilized landfill leachate onto micro- and meso-porous activated carbon. Water Res. 2012, 46, 491-499. [CrossRef] [PubMed]

16. Liu, Q.; Zhong, L.; Zhao, Q.; Frear, C.; Zheng, Y. Synthesis of $\mathrm{Fe}_{3} \mathrm{O}_{4}$ /Polyacrylonitrile Composite Electrospun Nanofiber Mat for Effective Adsorption of Tetracycline. ACS Appl. Mater. Interfaces 2015, 7, 14573-14583. [CrossRef] [PubMed]

17. Hsu, S.H.; Li, C.T.; Chien, H.T.; Salunkhe, R.R.; Suzuki, N.; Yamauchi, Y.; Ho, K.C.; Wu, K.C.W. Platinum-Free Counter Electrode Comprised of Metal-Organic-Framework (MOF)-Derived Cobalt Sulfide Nanoparticles for Efficient Dye-Sensitized Solar Cells (DSSCs). Sci. Rep. UK 2014, 4, 6983. [CrossRef] [PubMed]

18. Zhao, H.; Wang, Y.; Zhao, L. Magnetic Nanocomposites Derived from Hollow ZIF-67 and Core-Shell ZIF-67@ZIF-8: Synthesis, Properties, and Adsorption of Rhodamine B. Eur. J. Inorg. Chem. 2017, 35, 4110-4116. [CrossRef]

19. Ahmad, Z.U.; Yao, L.; Wang, J.; Gang, D.D.; Islam, F.; Lian, Q.; Zappi, M.E. Neodymium embedded ordered mesoporous carbon (OMC) for enhanced adsorption of sunset yellow: Characterizations, adsorption study and adsorption mechanism. Chem. Eng. J. 2019, 359, 814-826. [CrossRef]

20. Peng, B.; Chen, L.; Que, C.; Yang, K.; Deng, F.; Deng, X.; Shi, G.; Xu, G.; Wu, M. Adsorption of Antibiotics on Graphene and Biochar in Aqueous Solutions Induced by pi-pi Interactions. Sci. Rep. UK 2016, 6, 31920. [CrossRef] 
21. Zhu, Z.; Zhang, M.; Wang, W.; Zhou, Q.; Liu, F. Efficient and synergistic removal of tetracycline and Cu(II) using novel magnetic multi-amine resins. Sci. Rep. UK 2018, 8, 4762. [CrossRef] [PubMed]

22. Yu, F.; Ma, J.; Han, S. Adsorption of tetracycline from aqueous solutions onto multi-walled carbon nanotubes with different oxygen contents. Sci. Rep. UK 2014, 4, 5326. [CrossRef] [PubMed]

23. Zhou, Y.; Liu, X. Modification of biochar derived from sawdust and its application in removal of tetracycline and copper from aqueous solution: Adsorption mechanism and modelling. Bioresour. Technol. 2017, 245, 266-273. [CrossRef] [PubMed]

24. Zhang, X.; Shen, J.; Zhuo, N.; Tian, Z.; Xu, P.; Yang, Z.; Yang, W. Interactions between Antibiotics and Graphene-Based Materials in Water: A Comparative Experimental and Theoretical Investigation. ACS Appl. Mater. Interfaces 2016, 8, 24273-24280. [CrossRef] [PubMed]

25. Wang, Z.; Yu, X.; Pan, B.; Xing, B. Norfloxacin Sorption and Its Thermodynamics on Surface-Modified Carbon Nanotubes. Environ. Sci. Technol. 2010, 44, 978-984. [CrossRef]

26. Zhang, L.; Dong, D.; Hua, X.; Guo, Z. Inhibitory effects of extracellular polymeric substances on ofloxacin sorption by natural biofilms. Sci. Total Environ. 2018, 625, 178-184. [CrossRef]

27. Huang, P.; Ge, C.; Feng, D. Effects of metal ions and pH on ofloxacin sorption to cassava residue-derived biochar. Sci. Total Environ. 2018, 616-617, 1384-1391. [CrossRef]

(C) 2019 by the authors. Licensee MDPI, Basel, Switzerland. This article is an open access article distributed under the terms and conditions of the Creative Commons Attribution (CC BY) license (http://creativecommons.org/licenses/by/4.0/). 